\title{
SRCS: Statistical Ranking Color Scheme for Visualizing Parameterized Multiple Pairwise Comparisons with $\mathbf{R}$
}

\author{
by Pablo J. Villacorta and José A. Sáez
}

\begin{abstract}
The problem of comparing a new solution method against existing ones to find statistically significant differences arises very often in sciences and engineering. When the problem instance being solved is defined by several parameters, assessing a number of methods with respect to many problem configurations simultaneously becomes a hard task. Some visualization technique is required for presenting a large number of statistical significance results in an easily interpretable way. Here we review an existing color-based approach called Statistical Ranking Color Scheme (SRCS) for displaying the results of multiple pairwise statistical comparisons between several methods assessed separately on a number of problem configurations. We introduce an R package implementing SRCS, which performs all the pairwise statistical tests from user data and generates customizable plots. We demonstrate its applicability on two examples from the areas of dynamic optimization and machine learning, in which several algorithms are compared on many problem instances, each defined by a combination of parameters.
\end{abstract}

\section{Introduction}

When carrying out research in statistics, operational research and computer science, the problem of comparing a novel algorithm against other state-of-the-art techniques arises very often. The same idea can be applied to many other fields of science when introducing a new method for solving a well-known task, with the purpose of demonstrating the superiority of the proposed approach by numerically comparing the results with those obtained by already existing methods.

For some time now, it is widely accepted that statistical tests are required to compare several techniques that solve one given task (Demšar, 2006; García et al., 2010). This is motivated by the fact - also shown by Eugster et al. (2014) - that the performance of a technique for solving a task (for example, supervised classification) heavily depends on the characteristics of the concrete task instance (in this case, the data to which a classifier is to be fitted) and thus the experiments should randomize over a large number of datasets. Even with the same dataset, the results may vary when considering different subsets of training/test data (the former are used for fitting the model, and the latter for evaluating the model once it has been learned and does not change any more). The same applies to other very common machine learning tasks such as regression (Graczyk et al., 2010), approximate optimization using metaheuristics (García et al., 2009), and computational intelligence in general (Derrac et al., 2011). It should be noted that metaheuristics employed in optimization are by themselves randomized algorithms. Therefore, multiple runs of the same algorithm on the same optimization problem are required to assess an algorithm, as well as testing the performance over several different functions; we will further elaborate on this later. In order to analyze the results of these randomized trials, statistical tests are applied to draw a conclusion about the superiority of one method over the rest. A vast amount of literature exists dealing with this specific problem, see Coffin and Saltzman (2000); Shilane et al. (2008); García et al. (2010) and references therein, just to cite a few.

If one aims to visualize the results of statistical pairwise comparisons, the volume of data to display grows a lot if we take into account many problem configurations at the same time. The use of tables is very common as they summarize a lot of data in a compact way but they become hard to interpret when the results they contain are grouped in more than two parameters. It is usually very difficult to draw conclusions from big result tables, and for that reason, authors have developed data visualization techniques more sophisticated than boxplots or line charts, such as the figures presented in Demšar (2006) to distinguish between statistically different and indistinguishable algorithms, and other approaches explained in Bartz-Beielstein et al. (2010). A tool for the same purpose that is worth mentioning is the Model Viewer feature of the SPSS software (IBM Corp., 2012). When applied to hypothesis testing, it displays the multiple pairwise comparisons output as a complete graph where nodes represent the groups being compared, and arcs between them are colored differently according to the $p$-value of the corresponding comparison (in orange when the $p$-value is below a fixed significance threshold, and in black otherwise). Two remarkable tools are available for the R language. The paircompviz package (Burda, 2014), closely related to ours, makes use of Hasse diagrams with $p$-values in the arcs to represent the outcome of statistical tests. However, it does not use colors and it is not well suited for representing a large number of comparisons at once (as happens when we deal 
with many different problem configurations) since the resulting Hasse diagram would be too complex. The factorplot package recently published in this journal (Armstrong, 2013) focuses on hypothesis testing concerning the coefficients of generalized linear models or coefficients in multinomial logistic regression models, representing the results of the comparisons in grayscale grid plots. Our approach is more general and is oriented mainly to simulation-based studies.

Approximate optimization and machine learning constitute two areas of knowledge in which the problem of representing statistical results under several factors arises naturally. In both cases, we often want to compare the algorithm performance separately on different problem setups to highlight the conditions under which certain algorithms may work specially well. Existing studies in the field of dynamic optimization employ up to 40 numeric tables or graphs in a paper to summarize their results, due to the number of different experimental settings tested and the large amount of parameters involved in each problem configuration. Obviously, interpreting such a huge amount of numeric results becomes unfeasible. Moreover, none of the aforementioned visualization approaches deals well with multiple factor problems.

In order to solve this problem, a novel color-based technique for multiple pairwise statistical comparisons under several factors, called Statistical Ranking Color Scheme (SRCS), was introduced in del Amo and Pelta (2013) for comparing the performance of several dynamic optimization algorithms under a number of different problem configurations (del Amo et al., 2012). The method relies on a wise use of color scales that simplifies the identification of overall trends along many different problem settings simultaneously, thus enabling better understanding and interpretation of the results, and providing an overview of the circumstances under which each algorithm outperforms (or is outperformed by) the rest. However, no software package was available so far to automatically generate this kind of graphs at once from a dataset that collects the numerical results. The code published in del Amo and Pelta (2013) only calculates the ranking obtained by several algorithms on a fixed problem configuration, but does not plot the results nor allows for an automatic computation over a whole set of different problem configurations in order to obtain the images shown in del Amo et al. (2012).

Our aim here is to present an easy-to-use R package called SRCS (Villacorta, 2015) for creating fully customizable plots from a results file in experiments involving several factors, so that the user can configure how the plots should be arranged in the figure and has control over all graphical details of it, such as colors, fonts, titles, etc. Furthermore, we demonstrate the applicability of our package in two different contexts. The first is the comparison of algorithms to solve dynamic optimization problems (DOPs), which is the setting for which SRCS was originally conceived. The second is a novel application to machine learning tasks, where SRCS is used to compare the performance of several supervised classification algorithms over synthetic datasets created based on several parameters. Examples of these are noisy and/or imbalanced data for which parameters like the severity and type of noise, or the imbalance ratio are considered when generating the dataset from an originally clear one.

The remainder of this contribution is structured as follows. After the introduction the foundation of the SRCS technique and how multiple statistical significance results are displayed in color plots is reviewed. The next section presents an R package implementing SRCS, with a detailed description of the most important functions, their common uses and how they should be called. Then we explain two case studies where SRCS has been applied to visualize the statistical results of comparing a number of algorithms for two very different tasks, namely dynamic optimization and supervised classification when the data from which the classifier is learned contain noise or are imbalanced. Finally, the last section is devoted to conclusions and further work.

\section{Statistical ranking color scheme}

In this section we briefly review the foundations of SRCS (del Amo and Pelta, 2013). SRCS was developed for analyzing the relative performance of algorithms on a problem, rather than the absolute one. In other words, the outcome is a rank for each algorithm that depends on how many algorithms are better, equal or worse than the algorithm being ranked, where the decision on each pairwise comparison is given by a non-parametric statistical test over two sets of samples corresponding to multiple runs of each algorithm in exactly the same conditions. No distinction is made concerning the magnitude of the advantage or disadvantage in the performance comparison: SRCS is interested only in whether one algorithm is statistically better or worse than another, but not in how much.

The rank assigned to an algorithm $A_{i}$ on a problem configuration $c$ (determined by at most 3 parameters) is the sum of the scores obtained by the algorithm when comparing its performance $\left\{\operatorname{perf}_{k}\right\}_{i}^{c}, \quad k=1, \ldots, K$ against the rest of the algorithms (all pairwise comparisons) over the same problem configuration $c$. The performance is given by a sample composed by $K$ repeated observations obtained after $K$ independent runs of $A_{i}$ over the same problem configuration. It is assumed that 
either the nature of $A_{i}$ is itself randomized and gives a different output in each run, as happens with stochastic optimization algorithms, or the input data used by $A_{i}$ are a random sample and thus differ for each run, as happens for instance when using cross-validation (CV) for assessing a classification algorithm with a given dataset. In the $m$-fold CV method (typically $m=5$ or $m=10$ ), $m-1$ folds are used for building a model and the remaining fold is used for evaluating it and collecting the performance measure (accuracy or any other). This is repeated until every fold has been used exactly once as the test fold, hence collecting $m$ different performance values. If the complete $m$-fold $\mathrm{CV}$ process is repeated $r$ times, each time taking a different $m$-fold partition of the whole dataset, we obtain $K=m \cdot r$ independent measurements of the classifier's performance.

Ranks are calculated as follows. For each $j \neq i$, if the sample $\left\{\operatorname{perf}_{k}\right\}_{i}^{c}$ is statistically better (in the sense of the performance measure we are using) than $\left\{\operatorname{perf}_{k}\right\}_{j}^{c}$, then $A_{i}$ adds 1 point to its rank, and $A_{j}$ subtracts 1 point; if the opposite occurs, $A_{i}$ subtracts 1 point and $A_{j}$ adds 1 point. Otherwise, both algorithms are statistically equivalent so none of them modifies its rank. The initial rank of every algorithm is 0 . With this approach, when comparing $N$ algorithms, the maximum rank attainable by an algorithm is $N-1$, which means it outperforms the rest, and the minimum is $-(N-1)$, meaning it is outperformed by the rest.

The statistical test applied in pairwise comparisons could be customized by the user. In our implementation, we abide by the original proposal of del Amo and Pelta (2013) and use the pairwise Wilcoxon rank sum test with Holm's correction for multiple comparisons. Whether the test should be paired or not depends on the concrete problem we are facing, and can be set by the user. When assessing optimization algorithms, for instance, the test will most likely be non-paired since usually there is no relation between, say, the first execution of $A_{i}$ and the first execution of $A_{j}$ on the same problem configuration. In machine learning, the test should most likely be paired because all algorithms should be evaluated exactly with the same folds, hence the performance of the first execution of $A_{i}$ is paired with the first execution of $A_{j}$ because both were done with the same training and test subsets.

The strength of SRCS lies in its capability of arranging in a single plot the ranks obtained by many algorithms when tested separately over a lot of different problem configurations. Therefore, one can quickly visualize which configurations are the most favorable to each algorithm. This is done by using a grid of heatmaps. A heatmap represents three variables, namely the rank using a color scheme, and two variables in the $X$ and $Y$ axis of the heatmap, which we call the inner $X$ and $Y$ variables. At the same time, the whole heatmap is associated with one level of the other two variables, called the outer $\mathrm{X}$ and $\mathrm{Y}$ variables.

Figure 1 shows a toy example ${ }^{1}$ of ranking calculation and depiction of a simulated problem involving four algorithms that constitute the four levels of the outer Y variable. The problem involves three more variables, namely the outer $X$ variable (from which only the level outX1 is displayed), the inner $Y$ variable with four possible levels, and the inner $X$ variable with four possible levels as well. In Figure $1 \mathrm{c}$ the arrangement within the global plot is displayed for a concrete problem configuration that is allocated in the top left-most corner (as inner X variable $=1$, inner $Y$ variable $=4$ ) of the left-most column of heatmaps (since outer X variable $=$ outX1). The number of levels of all variables does not have to be the same as in this particular case.

\section{An R package implementing SRCS}

The aim of the SRCS package is to offer a set of functions to obtain figures similar to the one above in a straightforward manner and, at the same time, provide $\mathrm{R}$ users with full customization capabilities over graphical aspects like font size, color, axes aspect and so on. This has been accomplished by accepting tagged lists that are passed almost unchanged to some built-in graphical functions of the base package graphics on which our code relies. This package is very flexible and can be easily adapted so that the final plot has exactly the desired appearance. Package grid was also considered initially, but the adaptation would require more coding since the default aspect (more elegant) is slightly more complicated to fit our exact needs.

The general workflow can be summarized as:

1. Use function SRCSranks on the data being analyzed in order to compute the rank for each combination of factors according to the performance exhibited by that combination, following the rules explained in the preceding section.

2. Use function plot on the object returned by SRCSranks, indicating where each factor should be placed in the resulting plot, in order to obtain a color plot depicting the ranks calculated previously for all the factor combinations.

\footnotetext{
${ }^{1}$ Refer to Section Case studies for real examples.
} 

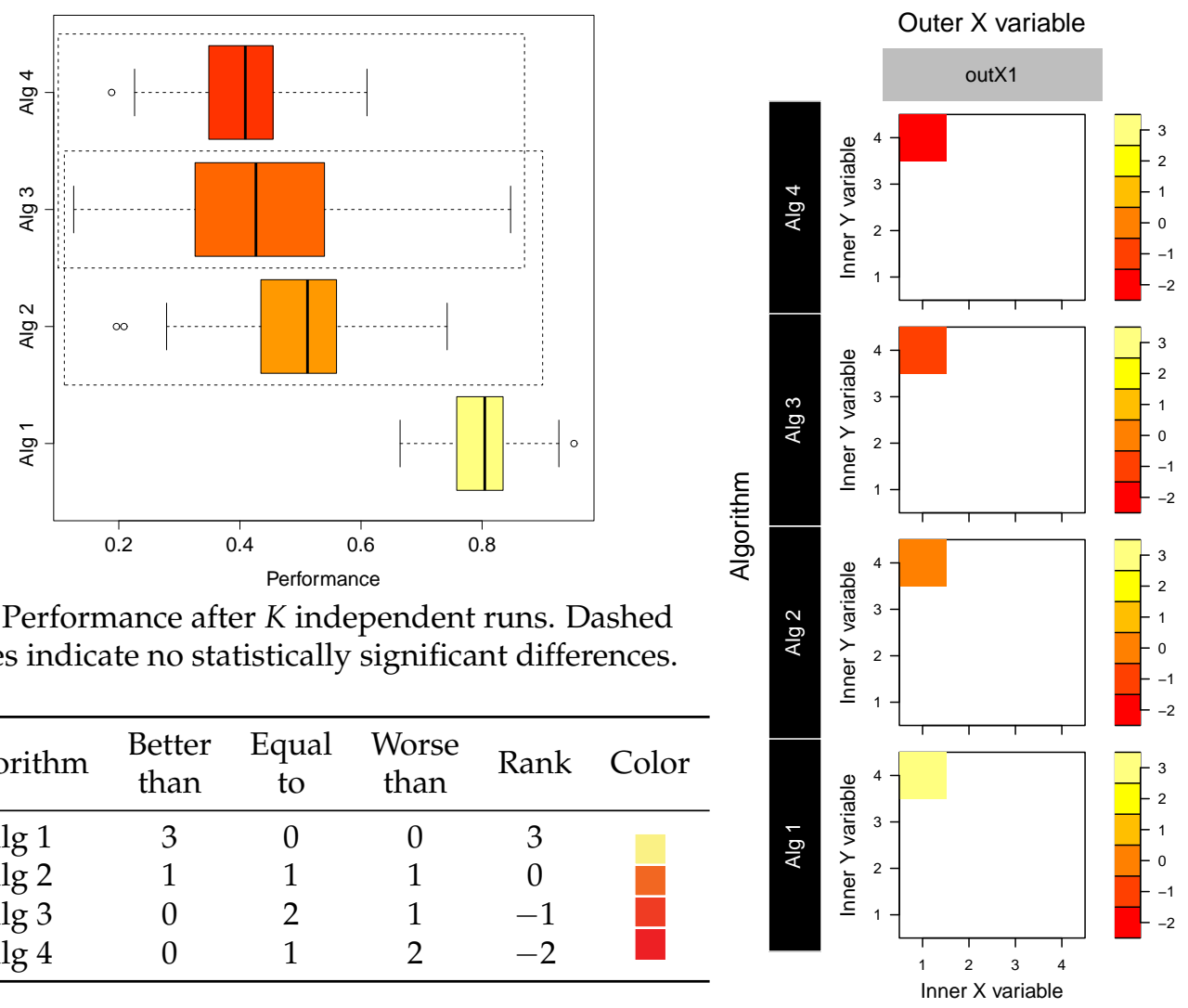

(a) Performance after $K$ independent runs. Dashed lines indicate no statistically significant differences.

\begin{tabular}{cccccc}
\hline Algorithm & $\begin{array}{c}\text { Better } \\
\text { than }\end{array}$ & $\begin{array}{c}\text { Equal } \\
\text { to }\end{array}$ & $\begin{array}{c}\text { Worse } \\
\text { than }\end{array}$ & Rank & Color \\
\hline Alg 1 & 3 & 0 & 0 & 3 & \\
Alg 2 & 1 & 1 & 1 & 0 & \\
Alg 3 & 0 & 2 & 1 & -1 & \\
Alg 4 & 0 & 1 & 2 & -2 & \\
\hline
\end{tabular}

(c) Arrangement in the plot.

(b) Rank calculation and color assigned.

Figure 1: Rank calculation of the relative performance of four algorithms in a problem configuration defined by Inner $X$ variable $=1$, Inner $Y$ variable $=4$, Outer $X$ variable $=$ outX1 .

3. (If needed) Use function SRCScomparison on the object returned by SRCSranks, specifying a concrete factor combination as well, to obtain a qualitative table describing the relative performance (measured from a statistical point of view) of every pair of levels of the target variable on the factor combination indicated. Each cell of the table contains a sign "=", ">" or " $<$ comparing the level on that row with the level on that column, where "=" stands for "no statistically significant differences found".

4. (If needed) Use function animatedplot on the object returned by SRCSranks, provided that the user data had more than one performance column, to visualize a video in which each video frame displays the ranks plot obtained by one performance column.

5. (If needed) Use function singleplot on the object returned by SRCSranks, specifying a factor combination that leaves two factors free, to visualize the ranks of one square of the full grid.

\section{Functions SRCSranks and SRCScomparison}

Our package exports five functions. Note that most of the arguments have default values to allow for a straightforward use if no customization is needed. The one that should be called first, prior to the plotting functions, is the following:

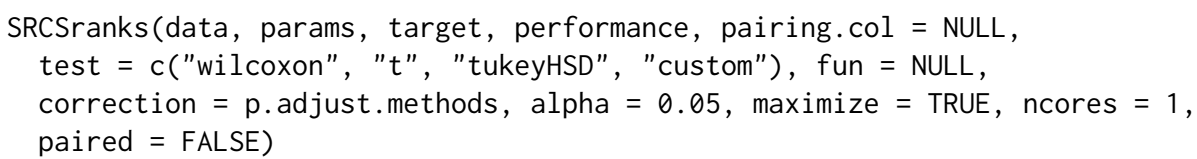

We review the meaning of the arguments below. For further details please refer to the corresponding help pages.

- data is a data frame containing the repeated performance measures together with their problem configuration (Table 1).

- params is a vector of strings with the names of the columns that define a problem configuration (here: $c(" A "$, " $B ", " C ")$ ). 


\begin{tabular}{cccccc}
\hline $\mathrm{A}$ & $\mathrm{B}$ & $\mathrm{C}$ & Target & Performance & Fold \\
\hline$a_{1}$ & $b_{1}$ & $c_{1}$ & Alg1 & 72.45 & 1 \\
$\vdots$ & $\vdots$ & $\vdots$ & $\vdots$ & $\vdots$ & $\vdots$ \\
$a_{1}$ & $b_{1}$ & $c_{1}$ & Alg1 & 72.36 & $\mathrm{~K}$ \\
\hline$a_{1}$ & $b_{1}$ & $c_{1}$ & $\mathrm{Alg} 2$ & 70.12 & 1 \\
$\vdots$ & $\vdots$ & $\vdots$ & $\vdots$ & $\vdots$ & $\vdots$ \\
$a_{1}$ & $b_{1}$ & $c_{1}$ & $\mathrm{Alg} 2$ & 69.89 & $\mathrm{~K}$ \\
\hline$a_{1}$ & $b_{1}$ & $c_{1}$ & $\mathrm{Alg} 3$ & 85.40 & 1 \\
$\vdots$ & $\vdots$ & $\vdots$ & $\vdots$ & $\vdots$ & $\vdots$ \\
$a_{1}$ & $b_{1}$ & $c_{1}$ & $\mathrm{Alg} 3$ & 85.21 & $\mathrm{~K}$ \\
\hline$\vdots$ & $\vdots$ & $\vdots$ & $\vdots$ & $\vdots$ & $\vdots$ \\
\hline
\end{tabular}

Table 1: A subset of the input data in a problem with a 3-level target variable, three problem-defining parameters $\mathrm{A}, \mathrm{B}$, and $\mathrm{C}$, with $\mathrm{K}$ observations of the performance per problem combination, and pairing between the samples. Showing only a fixed problem configuration defined by $\mathrm{A}=a_{1}, \mathrm{~B}=b_{1}, \mathrm{C}=c_{1}$. In this case, the column called Fold acts as the pairing column as it links the performance values within a given problem configuration for the paired statistical tests.

- target is the name of the target column whose levels are compared within each problem configuration (here, "Target").

- performance is the name of the column containing one or more observations of the response (or performance) variable associated to a problem configuration and a target level. It can be a string or a vector of strings, in which case the ranking process will be done independently for each of the performance columns indicated in the vector. This feature is used for composing videos showing the evolution of the performance at several time instants.

- pairing.col is the name of the column that indicates which performance values (corresponding to the same parameter configuration but different levels of the target variable) are linked with respect to the statistical tests. This value only makes sense if we set paired = TRUE; otherwise, it will be ignored.

- test is the statistical test (defaults to Wilcoxon) to be used for the pairwise comparisons (paired indicates whether a paired version of the test will be used or not). "custom" means a custom test will be applied, implemented by the function passed in the fun argument (which otherwise will be ignored).

- fun is a function implementing a custom statistical test for two samples that should return a tagged list with a p.values field, as occurs with pairwise.t. test and paired.wilcox. test, containing a matrix of $p$-values whose rows and columns have proper names.

- correction is the $p$-value adjustment method for multiple pairwise comparisons (defaults to Holm's procedure). It must be one of those natively implemented by $\mathrm{R}$ (ignored when test = "tukeyHSD").

- alpha is the significance threshold for the statistical test.

- maximize indicates whether the larger the performance, the better (default) or vice versa.

- ncores is the number of physical cores to be used in the computations. Parallelization is achieved through the function parLapply of the parallel package.

- paired indicates whether the multiple pairwise comparison tests should be paired or not (defaults to FALSE). When set to TRUE, the repeated performance observations are taken to be linked according to the values of the pairing. col column. For a given combination of params, the multiple observations associated to distinct levels of the target variable but sharing the same value of pairing.col are linked, as shown in column Fold of Table 1. Hence, all the pairwise comparisons between any two levels of the target variable will be paired.

The above function receives a data frame, chunks it according to all possible combinations of the values of params, and compares the levels of the target variable within each group by applying a statistical test to each binary comparison with the selected $p$-value adjustment method. When running in parallel, each processor manages a subset of all the chunks generated, where a chunk is composed 
of all the rows corresponding to a problem configuration. Therefore the input data are distributed among the processors by subsets of consecutive rows.

The output of the function is an object belonging to the S3 class 'SRCS' and extending class 'data. frame', which is actually a data frame containing all the params and target columns, a new rank column, two more columns with the average and the standard deviation of the performance for each problem combination, and additional columns summarizing the $p$-values of pairwise comparisons. In case more than one performance column was passed to the function, the output data frame will not contain the average, standard deviation and $p$-values columns, but just one rank column for each of the performance columns of the input data. The resulting object has been given an S3 class name 'SRCS' so that function plot can be applied on it after properly implementing a specific S3 method described below.

Function SRCScomparison receives the 'SRCS' object calculated by SRCSranks together with a problem configuration, and summarizes the $p$-values of the multiple pairwise comparisons. All the data are already present in the data frame returned by SRCSranks but not in an easily interpretable disposition. Therefore this function basically collects the $p$-values and prints them on screen in a nice way, either as a $p$-value table or showing only the qualitative result of every statistical comparison, i.e., $>,=,<$ for a fixed $\alpha$, without presenting the actual $p$-values. The function only works if the previous call to SRCSranks was done with only one performance column, because otherwise no $p$-values or average performances are calculated in the output data frame. The signature is the following:

SRCScomparison(rankdata, target, alpha $=0.05$, pvalues $=$ FALSE,$\ldots$ )

where rankdata is the data frame produced by SRCSranks, target is the name of the target column in rankdata, alpha is the significance threshold, pvalues indicates whether $p$-values or qualitative results of the comparisons should be printed, and ... is a succession of named arguments corresponding to columns of rankdata and their values to fully determine a problem configuration. These named arguments are used for subsetting rankdata. The number of rows of this subset should be equal to the number of levels of the target variable; otherwise an error is thrown.

\section{The S3 plot method for 'SRCS' objects}

The data frame produced by SRCSranks is usually passed on to the next function, which is the S3 plot method for 'SRCS' objects and constitutes the main component of the package:

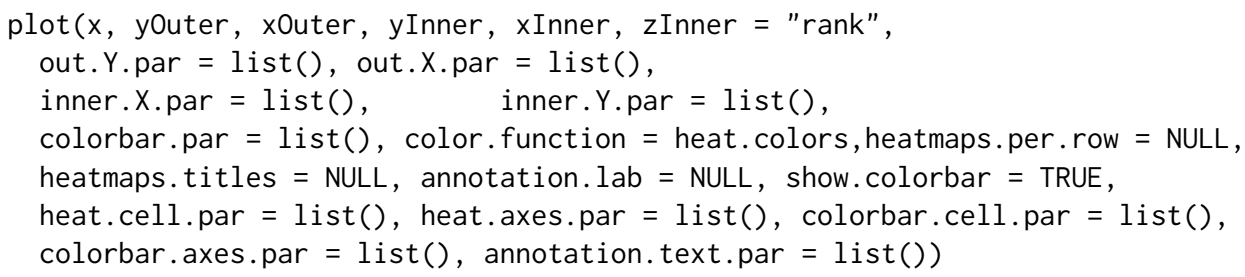

Below we provide a brief description of all the parameters. For further details please refer to the package help pages. Notice only the first five arguments are mandatory.

- $\mathrm{x}$ is an 'SRCS' object usually generated by a call to SRCSranks but can also be directly composed by the user. This way, the user can create his own ranks and use the SRCS package only to plot them, as long as they are properly arranged in a data frame with class 'SRCS' as those generated by SRCSranks.

- yOuter, xOuter, yInner, xInner, zInner are the names of the columns that will be plotted in each of the dimensions of the plot; see Figure 1c, where the Algorithm plays the role of the outer $\mathrm{Y}$ variable. The zInner variable corresponds to the rank column, which is plotted using colors in the heatmaps: the higher the value of zInner, the better, and hence, the lighter the color assigned to it. The location of the levels both in the outer and inner variables depends on the factor levels for these variables when transforming them to factors, a conversion that takes place inside the function.

- out.Y.par, out.X.par, inner.X.par, inner. Y. par are tagged lists to customize how variable labels and level labels are displayed. Some options include hiding a label, setting the character size, color, location, orientation, whether it should be placed inside a rectangle or not, border and background color of such a rectangle, and other parameters that will be passed directly to the text function in the graphics package. Arguments heat.cell.par, heat.axes.par, colorbar.cell. par, colorbar. axes. par and annotation. text.par play a similar role.

- color. function is a function returning a vector of hexadecimal color codes of length (maxrank - minrank +1 ) which will be used for displaying the heatmaps. Can be either a custom 
function or one of the existing palettes such as heat.colors, terrain. colors, etc. The function will be called with one argument, namely the number of colors to be returned, (maxrank minrank +1$)$.

- heatmaps.per.row is an integer indicating whether all the levels of the outer $\mathrm{X}$ variable are shown horizontally, or broken in two or more sub-rows.

- show. colorbar is a Boolean that displays or hides a colorbar used as the legend of the figure.

- heatmaps. titles is a vector of strings with the titles of every individual heatmap, if needed.

- annotation. lab is an annotation string that will be displayed on the top left corner of the plot. This is useful for labeling individual plots when composing videos.

The function relies on the layout function of the graphics package to automatically compose a suitable layout, taking into account the number of levels of each variable and the user's choices.

\section{Functions animatedplot and singleplot}

Function animatedplot enables composing videos from sequences of plots like Figure 3. This enables the user to visualize time as a new dimension by plotting statistical pairwise comparison results at different time moments. This can be useful, for instance, when comparing convergence speed between many algorithms about which the best solution so far has been annotated at different moments of the optimization process. The function relies on R's built-in capability to automatically compose counterbased filenames when successively generating plots to image files, and then calls ImageMagick (Still, 2005), a widely used open-source software for Windows and Linux, to join them together into a video file. A number of image formats can be used for the images generated prior to composing the video. Note that those files are not automatically deleted; the user will have to do it by himself. It is necessary that the user has previously installed ImageMagick.

The function signature is the following:

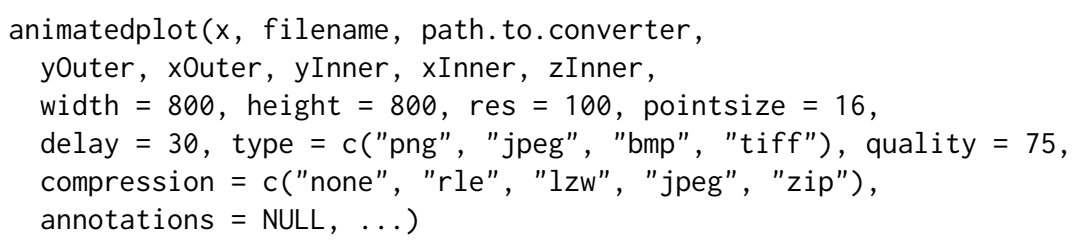

In this case, $z$ Inner should be a vector with the names of the columns in $x$ containing the performance measures to be plotted successively. The video will have as many frames as elements there are in zInner. The argument path. to. converter is a string with the full path of the converter program that comes with ImageMagick, e.g., "C: /Program Files/ImageMagick-<version>/convert. exe". The rest of the arguments allow setting the name of the output video file (including the file format) and configure the size, resolution, delay between the frames (in 1/100th of a second), percentage of quality and type of compression. The function also gives the possibility to set an independent annotation in the upper-left corner of each frame by passing a vector of strings, where each element is the annotation of the corresponding frame of the sequence. The ... argument accepts any subset of the optional arguments to be passed to the S3 plot method for 'SRCS' objects that plots every frame.

Function singleplot creates a single heatmap focused on the problem configuration defined by the user. It has the following signature:

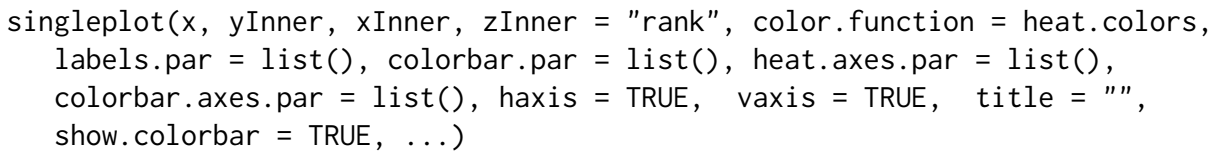

The parameters are similar to those already described. The ... argument in this case stands for a succession of named arguments (as many as necessary) that will be used to subset the data argument. From that subset, the values of the zInner column will be depicted in a single heatmap, in the locations indicated the by yInner and xInner columns. If any pair of values of columns ( $x$ Inner, yInner) is found more than once after subsetting, an error is thrown.

\section{Case studies}

In this section, two examples representative of those typically faced by potential users will be presented, with the purpose of illustrating the package capabilities and ease of use. None of them is aimed at finding the best algorithm for the posed problems, but at showing the applicability of the package 
in different areas of knowledge when analyzing experimental results. Therefore, the details of the experimental framework in each example (such as the tuning of the parameters, the concrete algorithms and datasets tested and so on) are not relevant for the aforementioned purpose ${ }^{2}$.

The first example analyses the results of dynamic optimization algorithms while the second deals with typical machine learning problems where several classification algorithms are compared under different settings or problem configurations. Note that the package is oriented at the analysis of experimental results, which do not necessarily come from $\mathrm{R}$ code or even from a computer program. In our case, the techniques assessed in the first example have been implemented in Java and are not available in $\mathrm{R}$.

\section{Application to dynamic optimization problems}

DOPs (Branke, 2001) are a variant of classical optimization problems in which the function being optimized has time-dependent properties, i.e., changes along the time during the execution of the optimization algorithm itself. The changes may affect the fitness function, the constraints, the number of variables of the function or their domain, etc. DOPs have attracted increasing attention due to their closeness to many real-world changing problems, as explained in the aforementioned work.

Many algorithms have been proposed to solve DOPs as explained in Cruz et al. (2011), most of them based on Evolutionary Algorithms and other population-based metaheuristics. Here we will reproduce one of the plots published in del Amo et al. (2012) representing a broad DOP algorithm comparison, including the $\mathrm{R}$ code necessary to obtain them in a straightforward way. The numerical results represented in the plots have been included as a data frame object called MPB in the SRCS package. Details on the algorithms compared can be found in the aforementioned work. Below we briefly comment on the meaning of the parameters involved in a problem configuration, the performance measure collected in the file and the fitness function we are optimizing.

In a DOP, the fitness function changes along the time. Several aspects modulate how this happens, such as the time passed between two consecutive changes, or the severity of the change (how different the function is with respect to the previous version). None of these parameters is known in advance by any algorithm. The third parameter known to affect the performance is the dimension of the function, which is user-configurable but remains invariant during the execution.

The fitness function employed, known as the Moving Peaks Benchmark (MPB, Branke 1999; see Figure 2a), was specifically designed as a DOP benchmark. The problem consists in maximizing a continuous $n$-dimensional function that results from the superposition of $m$ peaks, each one characterized by its own height $\left(h^{j} \in \mathbb{R}\right)$, width $\left(w^{j} \in \mathbb{R}\right)$ and location of its centre $\left(p^{j} \in \mathbb{R}^{n}\right)$ :

$$
\operatorname{MPB}(\mathbf{x})=\max _{j}\left\{h^{j}-w^{j} \sqrt{\sum_{i=1}^{n}\left(x_{i}-p_{i}^{j}\right)^{2}}\right\}, \quad j=1, \ldots, m .
$$

The global optimum is the centre of the peak with the highest parameter $h^{j}$. To make this function dynamic, the parameters of the peaks are initialized to some prefixed values, but then change every $\omega$ function evaluations according to certain laws (refer to Branke 1999; del Amo et al. 2012 for details). The values of the parameters used in the experiments are summarized in Figure 2b. The first three rows can vary to define every single problem configuration, while the rest are fixed for all problem configurations.

A lot of different performance measures have been proposed for DOPs as mentioned in Cruz et al. (2011). Here we employ the most widely accepted one, namely the offline error (del Amo et al., 2012):

$$
e_{o f f}=\frac{1}{N_{c}} \sum_{i=1}^{N_{c}} \frac{1}{N_{e}(i)} \sum_{j=1}^{N_{e}(i)}\left(f_{i}^{*}-f_{i j}\right)
$$

where $N_{c}$ is the total number of changes in the environment during the execution, $N_{e}(i)$ is the total number of evaluations allowed in the $i$-th change, $f_{i}^{*}$ is the optimum value of the $i$-th change, and $f_{i j}$ is the best value found by the algorithm since the beginning of the $i$-th change up to the $j$-th evaluation. It is defined this way to favor those algorithms which converge to good solutions very quickly after each change. Furthermore, since changes take place at a fixed rate in our experiments $\left(N_{e}\left(i_{1}\right)=N_{e}\left(i_{2}\right)=\ldots=N_{e}\right)$, the formula simplifies to

$$
e_{o f f}=\frac{1}{N_{c} N_{e}} \sum_{i=1}^{N_{c}} \sum_{j=1}^{N_{e}}\left(f_{i}^{*}-f_{i j}\right) .
$$

\footnotetext{
${ }^{2}$ This section has been expanded in the package vignette with a third case study.
} 


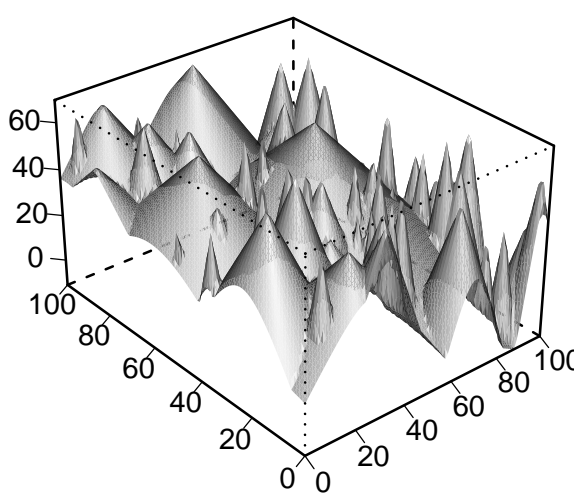

(a) The MPB function in two variables.

\begin{tabular}{ll}
\hline Parameter & Values tested \\
\hline Dimension $(n)$ & $\{5,10,15,20,25\}$ \\
Change period $(\omega)$ & $\{40,100,200, \ldots, 1000\} \cdot n$ \\
Severity $(s)$ & $\{2 \%, 4 \%, \ldots, 20 \%\} \cdot x$ range \\
\hline$x$ range & {$[0,100]$ for every variable } \\
Correlation coef. $(\lambda)$ & 0.5 \\
Number of peaks $(m)$ & 100 \\
Peak heights $\left(h^{j}\right)$ & $\mathcal{U}[30,70]$ \\
Peak widths $\left(w^{j}\right)$ & $\mathcal{U}[1,12]$ \\
Height severity $\left(h_{s}\right)$ & 7.0 \\
Width severity $\left(w_{s}\right)$ & 1.0 \\
\hline
\end{tabular}

(b) Parameters used in the MPB experiments.

Figure 2: Experimental setup in the MPB.

As this is a maximization problem, $f_{i}^{*}-f_{i j}$ remains always positive. For each problem configuration \{change period, severity, dimension\}, every algorithm is run $K=50$ independent times, thus collecting 50 offline error measurements which will be used to perform the pairwise statistical tests. In each run, the fitness function changes $N_{c}=100$ times along the execution, at regular time intervals.

The $\mathrm{R}$ code used to plot the results is the following:

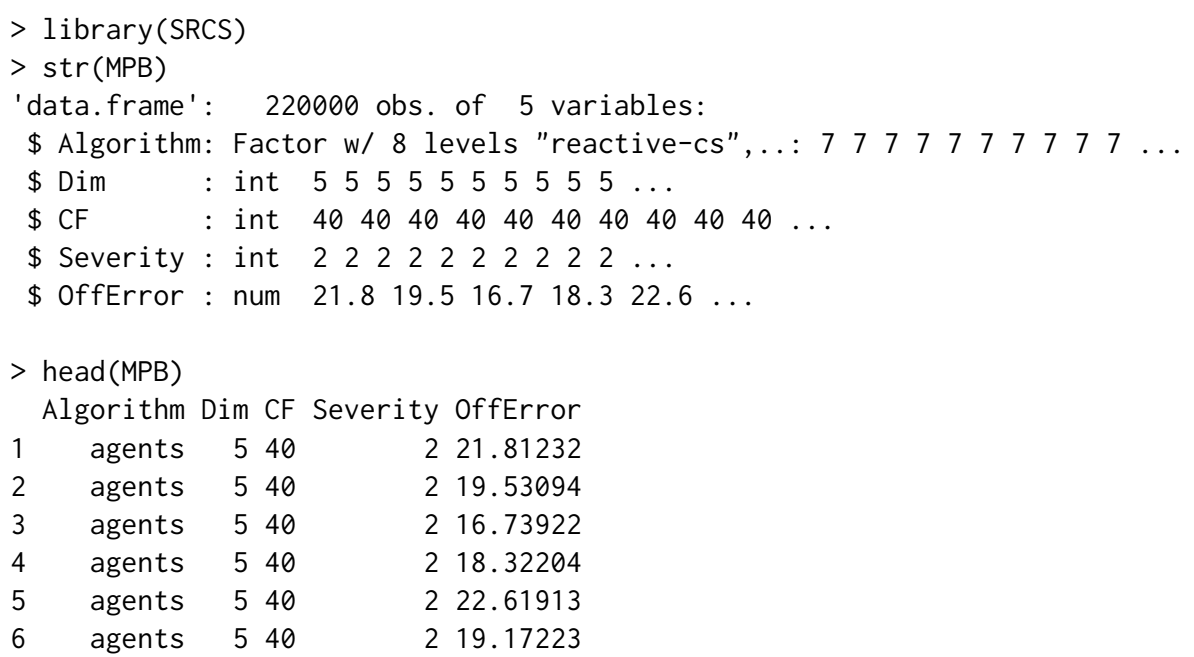

The above output is the first part of the 50 performance observations of algorithm agents in the problem configuration defined by Dim $=5, C F=40$, Severity $=2$. Note that the tests should be non-paired as there is no relation between the runs of the algorithms.

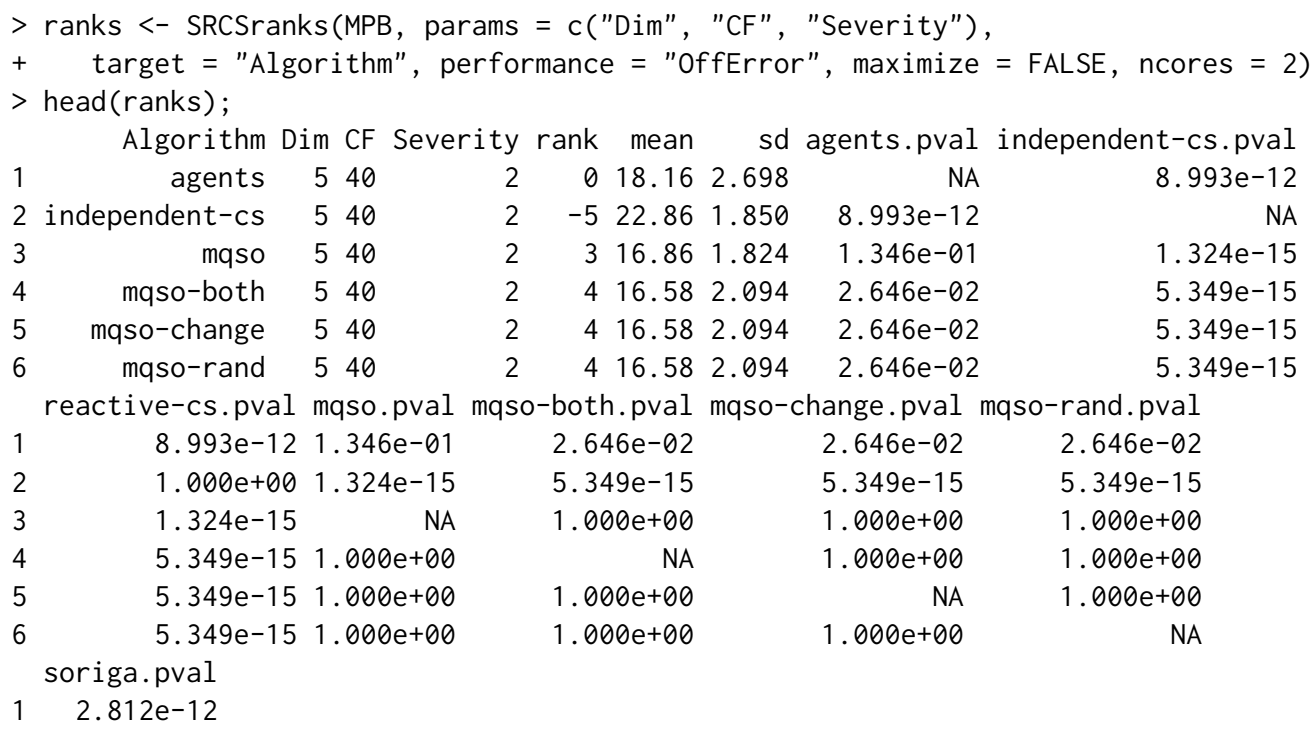


$2 \quad 1.466 \mathrm{e}-01$

$3 \quad 4.419 \mathrm{e}-15$

$4 \quad 5.349 \mathrm{e}-15$

$5 \quad 5.349 \mathrm{e}-15$

$6 \quad 5.349 \mathrm{e}-15$

Note that the $K$ rows per problem configuration present in the input data have now collapsed into one row per problem configuration, containing the average performance of the $K$ observations, their standard deviation, the rank achieved by that configuration, and the corrected $p$-values of the multiple pairwise statistical tests against the other levels of the target variable in the same problem configuration. We can now plot these results with the following code:

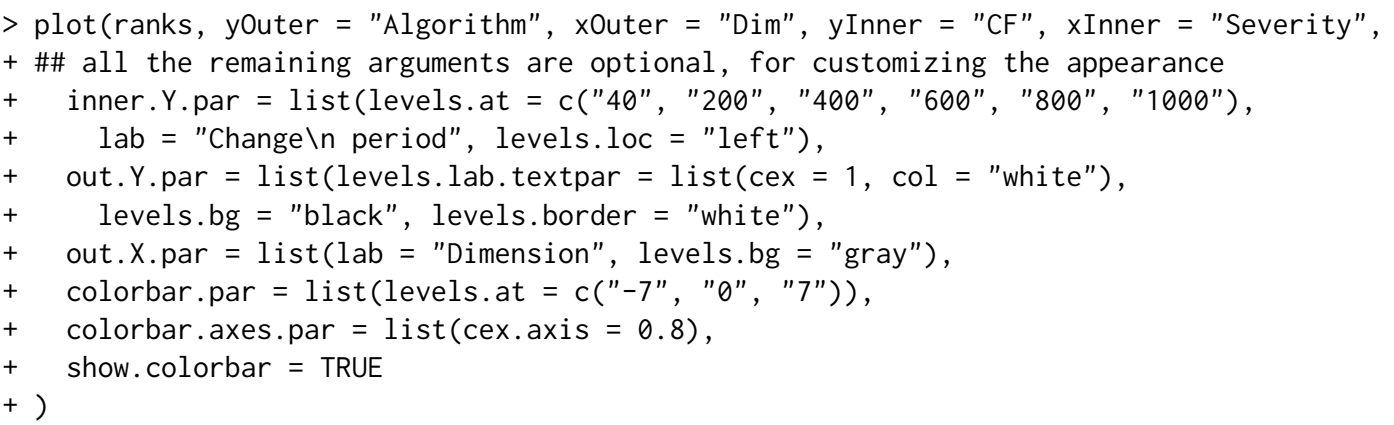

The results are depicted in Figure 3, which should be interpreted as follows: for a given value of Dimension, one should look at the whole column of heatmaps vertically to know how the algorithms behave for that dimension. The arrangement of the cells within the heatmaps is analogous to Figure 1c. From the figure, we can see that, for instance, soriga only behaves well (although it is not the best one) when the change period is short, and this is enhanced when increasing the dimensionality of the problem. This amounts to say that soriga is specially good at detecting a change and recovering from it by quickly discovering promising regions after the change, although it is not so good at exploiting these regions (it is beaten by other algorithms when the change period gets larger). On the other hand, agents also improves its behaviour when the dimensionality grows above 15 (otherwise, mqso-rand dominates the rest when considering a 5- or 10-variable fitness function), but also when severity increases, becoming the best algorithm in those cases (right part of the heatmaps).

We could ask for a single heatmap as well, defined by some values of the outer $\mathrm{Y}$ and $\mathrm{X}$ variables, for instance Algorithm = soriga and Dimension = 25, using the following call:

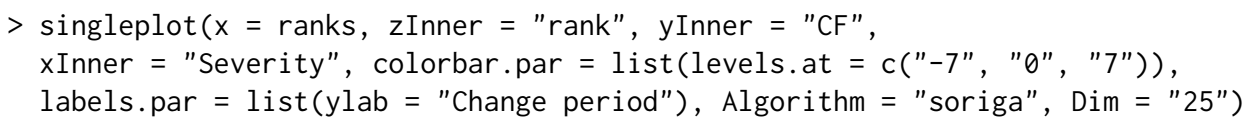

The output is shown in Figure 4. To obtain a qualitative performance comparison for a given problem configuration, for instance when Change period $=40$, Dimension $=25$, Severity $=20$, we can use the following call:

SRCScomparison(ranks, "Algorithm", CF $=40$, Dim $=25$, Severity $=20$, pvalues $=$ FALSE)

which will produce the following matrix object as a result:

\begin{tabular}{|c|c|c|c|c|c|c|c|c|}
\hline & agents & indep-cs & mqso & mqso-both & mqso-change & mqso-rand & reactive-cs & soriga \\
\hline agents & $\mathrm{NA}$ & "<" & $"<"$ & "<" & "<" & "<" & "<" & "<" \\
\hline indep-cs & ">" & NA & $"<"$ & "<" & $"<"$ & $"<"$ & $"="$ & ">" \\
\hline mqso & ">" & ">" & NA & ">" & $"="$ & ">" & $">"$ & ">" \\
\hline mqso-both & $">"$ & $">"$ & $"<"$ & NA & $"<"$ & $"="$ & $">"$ & ">" \\
\hline mqso-change & $">"$ & $">"$ & $"="$ & $">"$ & NA & $">"$ & ">" & ">" \\
\hline mqso-rand & $">"$ & ">" & $"<"$ & $"="$ & $"<"$ & NA & ">" & ">" \\
\hline reactive-cs & $">"$ & $"="$ & $"<"$ & $"<"$ & $"<"$ & $"<"$ & NA & ">" \\
\hline soriga & $">"$ & $"<"$ & $"<"$ & $"<"$ & $"<"$ & $"<"$ & $"<"$ & NA \\
\hline
\end{tabular}

Composing a video file to visualize convergence In the vignette associated to this package it is shown how to create a video to visualize an extra temporal component in the results. The data were collected from the aforementioned executions of dynamic optimization algorithms over the MPB problem, annotating the offline error at the instant before every change. Both the data, the R script and the resulting video can be downloaded from the first author's personal home page ${ }^{3}$, which is also given as URL in the package description. Please refer to the vignette for further details.

${ }^{3}$ http://decsai.ugr.es/ pjvi/r-packages.html 


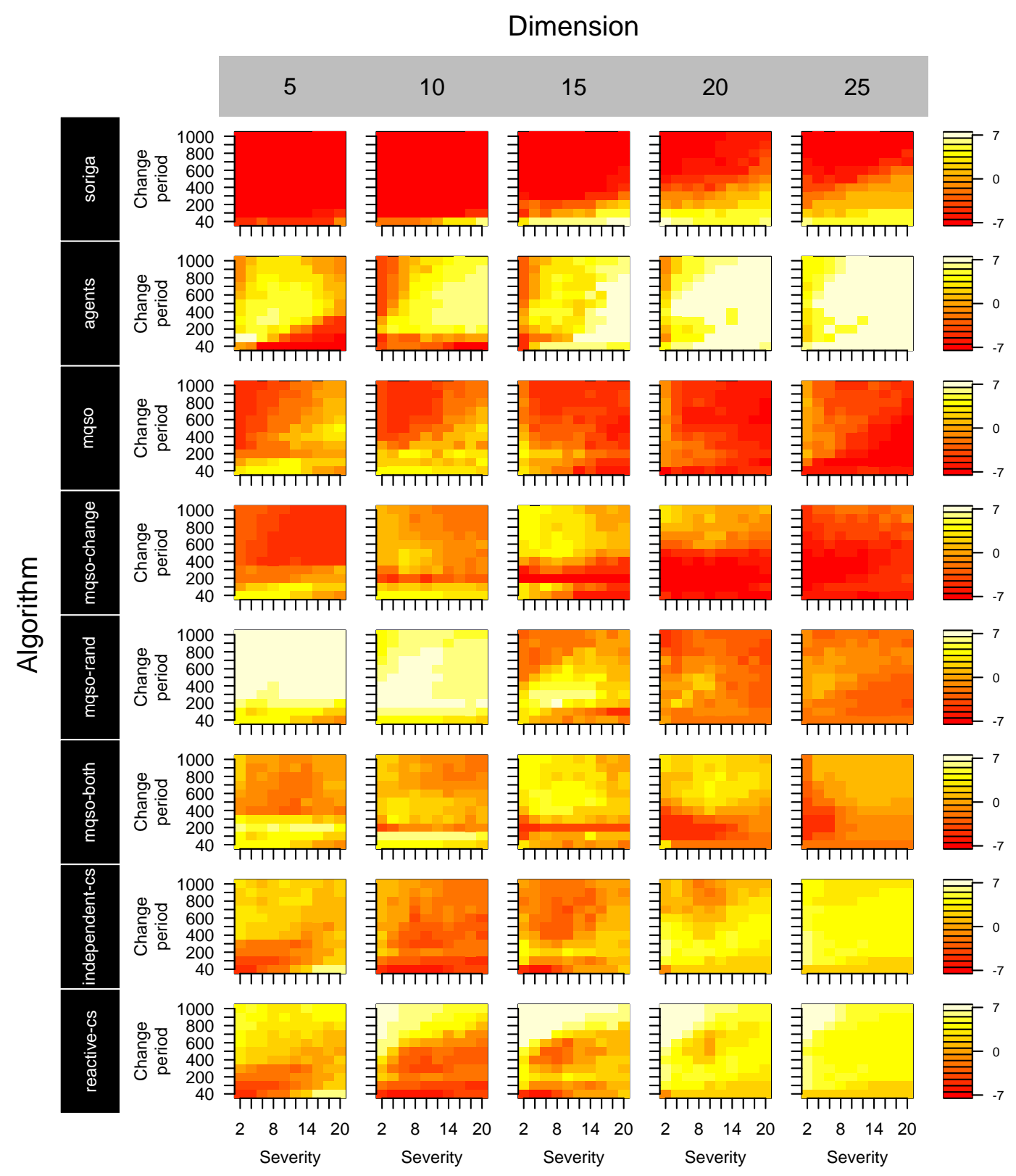

Figure 3: Results of several dynamic optimization algorithms on the MPB. This plot mirrors Figure 5 of del Amo et al. (2012). 


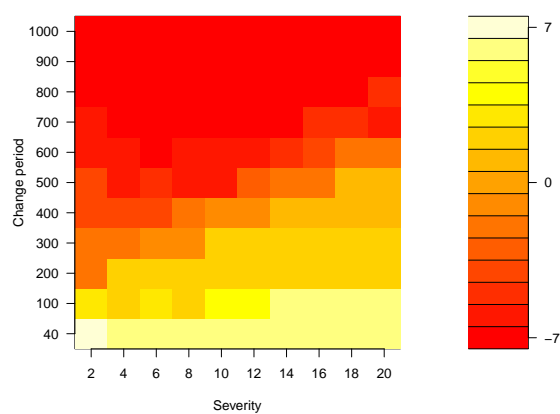

Figure 4: A single heatmap generated by singleplot for problem configurations where Algorithm= soriga, Dimension $=25$.

\begin{tabular}{lccclccc}
\hline Dataset & \#EX & \#AT & \#CL & Dataset & \#EX & \#AT & \#CL \\
\hline automobile & 159 & $25(15 / 10)$ & 6 & glass & 214 & $9(9 / 0)$ & 7 \\
balance & 625 & $4(4 / 0)$ & 3 & ionosphere & 351 & $33(33 / 0)$ & 2 \\
cleveland & 297 & $13(13 / 0)$ & 5 & pima & 768 & $8(8 / 0)$ & 2 \\
ecoli & 336 & $7(7 / 0)$ & 8 & vehicle & 846 & $18(18 / 0)$ & 4 \\
\hline
\end{tabular}

Table 2: Description of the classification datasets.

\section{Application to machine learning: Noisy datasets}

The second case study is a machine learning experiment involving six supervised classification algorithms tested over a number of noisy datasets with several types of noise and noise severities. The aim is to assess how noise affects each of the classifiers and whether the behaviour changes with those parameters. The algorithms tested were the following: (a) the $k$-Nearest-Neighbours classifier with three different values of $k(k=1, k=3$ and $k=5)$, (b) Repeated Incremental Pruning to Produce Error Reduction (RIPPER), (c) a Support Vector Machine (SVM), and (d) the C4.5 tree-based rule induction classifier. The reader may refer to Cohen (1995); Bishop (2006) for a review of all these algorithms.

$\mathrm{R}$ implementations have been used in all cases. We coded the $k$-NN to incorporate the HVDM distance (Wilson and Martinez, 1997) for heterogeneous (continuous and nominal) attributes. The SVM was taken from the e1071 package, version 1.6-4 (Meyer et al., 2014), which contains a wrapper for the libsvm C++ library (Chang and Lin, 2001). Algorithms C4.5 and RIPPER were taken from RWeka, version 0.4-24 (Hornik et al., 2009), which offers an R interface to the Weka framework (Witten and Frank, 2005). The datasets employed in the experiment (Table 2) have been taken from the UCI repository (Lichman, 2013), and are among the most widely used in machine learning studies. For each dataset, the number of classes (\#CL), the number of examples (\#EX) and the number of attributes (\#AT), along with the number of numeric and nominal attributes are presented.

In the literature, two types of noise can be distinguished in a dataset (Zhu and Wu, 2004): (i) class noise (examples labeled with a class distinct from the true one) and attribute noise (that usually refers to erroneous attribute values). The amount and type of noise present in real-world datasets are usually unknown. In order to control the amount of noise in the datasets and check how it affects the classifiers, noise is introduced into each dataset in a controlled manner. Four different noise schemes have been used in order to introduce a noise level $x \%$ into each dataset (Zhu and $\mathrm{Wu}, 2004)$ :

\section{Introduction of class noise.}

- Random class noise (CLA_RAND). $x \%$ of the examples are randomly selected and turned corrupt. The class labels of these examples are randomly replaced by another one from the $M$ classes.

- Pairwise class noise (CLA_PAIR). Let $X$ be the majority class and $Y$ the second majority class. An example with the label $X$ has a probability of $x / 100$ of being incorrectly labeled as $Y$.

\section{Introduction of attribute noise.}

- Random attribute noise (ATT_RAND). $x \%$ of the values of each attribute in the dataset are randomly selected and turned corrupt. To corrupt each attribute $A T_{i}, x \%$ of the examples in the dataset are chosen, and their $A T_{i}$ value is replaced by a random value from the 
domain $\mathbb{D}_{i}$ of the attribute $A T_{i}$. An uniform distribution is used for both numerical and nominal attributes.

- Gaussian attribute noise (ATT_GAUS). This scheme is similar to the uniform attribute noise, but in this case, the $A T_{i}$ values are corrupted, adding a random value to them following a Gaussian distribution of mean $=0$ and standard deviation $=(\max -\min ) / 5$, being $\max$ and min the limits of the attribute domain $\left(\mathbb{D}_{i}\right)$. Nominal attributes are treated as in the case of the uniform attribute noise.

The four noise schemes have been considered independently and for each type of noise, the noise levels ranging from $x=0 \%$ (base datasets) to $x=50 \%$, by increments of $5 \%$, have been studied. The accuracy estimation of the classifiers in a dataset is obtained by means of a stratified 5-fold crossvalidation, which is the standard in the field. For obtaining multiple observations, the cross-validation procedure was repeated five times, thus obtaining $K=25$ performance (accuracy rate) values for each algorithm in each problem configuration, defined by \{dataset, noise type, noise severity\}. These values will later be used in pairwise statistical comparisons. For a given problem configuration, exactly the same partitions of a dataset were used with all the algorithms, and for that reason, the observations are paired (recall Table 1 ).

Performing pairwise comparisons separating the results by dataset can be particularly useful in certain machine learning works which include a very small number of datasets. In those studies, the conventional approach consisting in summarizing the performance of an algorithm over a dataset with a single value and applying post-hoc pairwise comparisons between the algorithms with these summaries does not work, because each of the samples being compared has too few elements (due to the reduced number of datasets) to apply a statistical test. In such cases, the SRCS approach would be more suitable and would yield a reliable comparison for each dataset separately.

The R script which runs the algorithms over the datasets mentioned and generates the results to be analyzed can be downloaded from the first author's home page mentioned before, together with the datasets. The performance results obtained are already included in the package, to save time. When SRCS is loaded, a data frame object called ML1 containing the results of this experiment is created:

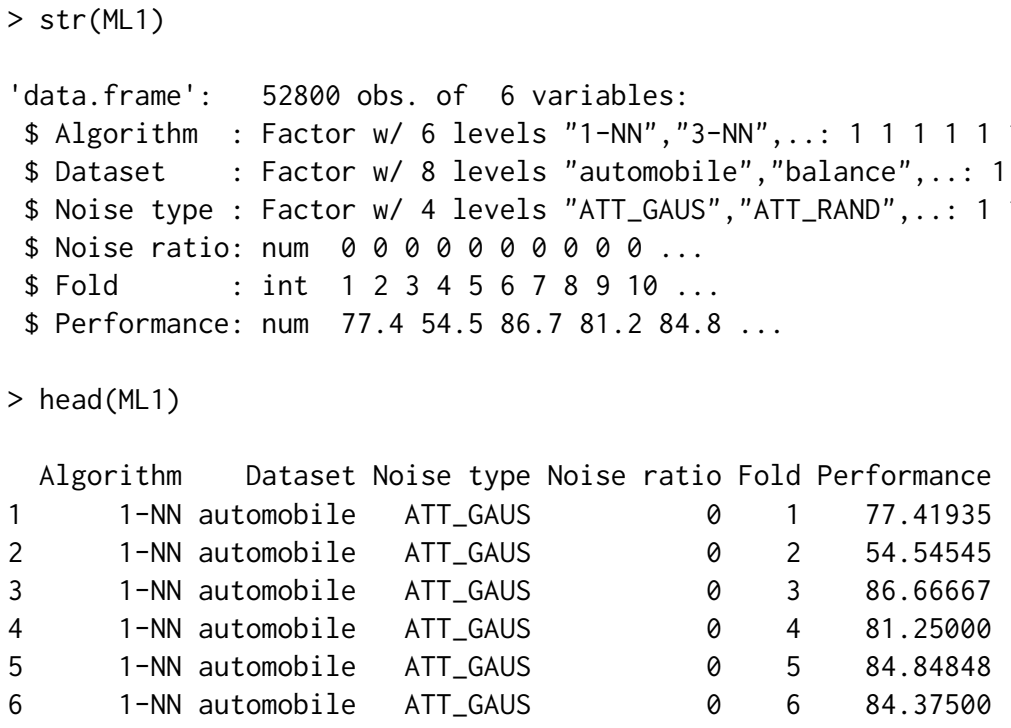

The R code to compute and plot the ranks with SRCS is the following.

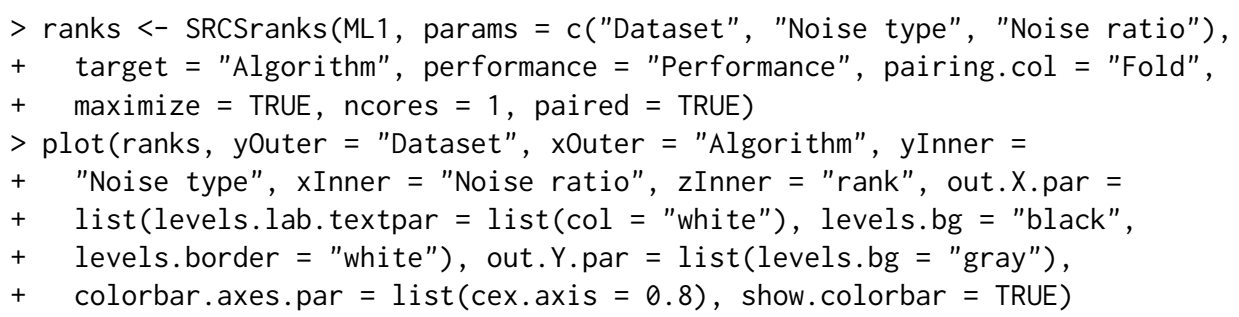

The results are summarized in Figure 5. This figure shows that higher values of $k$ in the $k$-NN classifier make the model perform better than lower values of $k$ (with the exception of the automobile dataset, where the opposite happens). Thus, 5-NN generally is better than 3-NN, and 3-NN is better than $1-\mathrm{NN}$ for the different datasets considered. This fact is in accordance with the work of Kononenko and Kukar (2007) that claimed that the value of $k$ in $k$-NN determines a higher or lower sensitivity to 


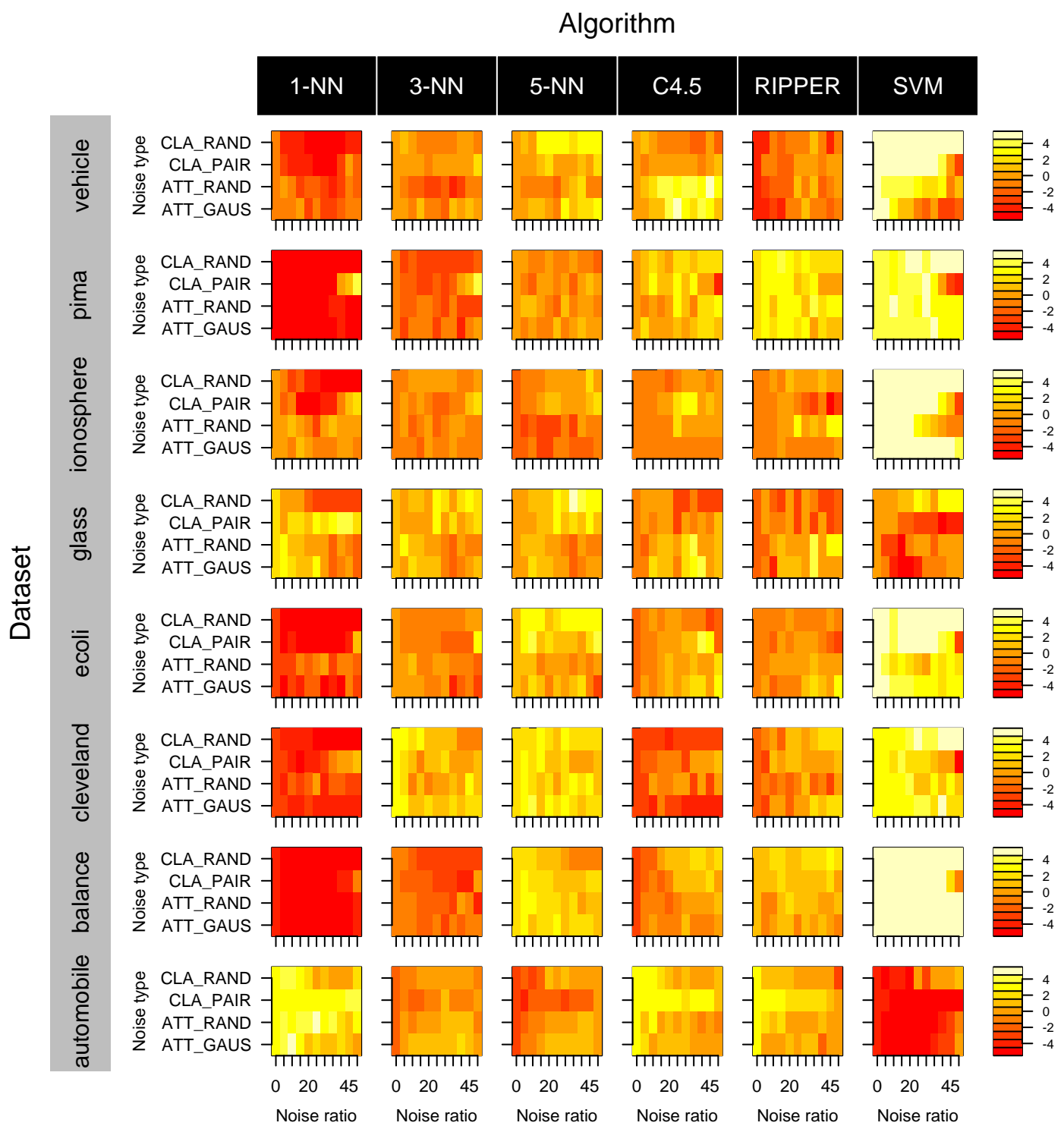

Figure 5: Results of six supervised classification algorithms on eight noisy datasets.

noise. SVM presents variable results, depending on the dataset analyzed. For some of them, such as automobile or glass, the results are predominantly in red colours. Other datasets, such as vehicle or cleveland, show that SVM can work relatively well when the noise level is low, but its performance is deteriorated when the noise level increases. These facts agree with the results of the literature that state that SVM is usually noise-sensitive, particularly with high noise levels (Nettleton et al., 2010). However, for other datasets considered, such as balance, SVM obtains good results. Finally, one must note that both C4.5 and RIPPER, which are considered robust to noise (Zhu and Wu, 2004), obtain intermediate results in the eight datasets considered.

\section{Conclusions and further work}

In this paper we have introduced an R package called SRCS, aimed at testing and plotting the results of multiple pairwise statistical comparisons in different configurations of a problem, defined by several parameters. The package implements a previously published visualization technique to summarize the output of many comparisons at the same time by using a careful spatial arrangement to display the result for each problem configuration defined by a parameter combination. As we have explained, our code gives the user full control over all the graphical options so as to fully customize the plot. Furthermore, we have taken this approach a step further by considering the time as another parameter. This turns static images into videos to take into account this new dimension, but allows constructing convergence plots for all problem configurations simultaneously. It should be noticed that, while videos have been conceived to represent convergence, they can also be used with another variable in 
any setting in which it makes sense to watch the evolution of statistical results.

We have successfully applied our package to two very different problems, namely dynamic optimization problems and machine learning problems. The latter represents a novel use of SRCS that has proven very helpful for comparing classification algorithms under different circumstances of noise type, noise levels, imbalance ratios and shape of the data. The SRCS approach enables visualizing the results of a number of algorithms at a glance, which in turns leads to an easier interpretation and may also reveal trends relating different problem configurations that otherwise would be harder to uncover, such as the configurations where each algorithm (or family of algorithms) performs best.

An interesting improvement would consist in adding interactivity to the plots. The user could manually re-arrange the plots or add/remove problem parameters and/or target levels, and visually check whether such modifications cause a strong change in the results or not as the plot would be automatically updated.

\section{Acknowledgments}

This work is supported by projects TIN2011-27696-C02-01 from the Spanish Ministry of Science and Innovation, P11-TIC-8001 from the Andalusian Government, and FEDER funds. P. J. Villacorta acknowledges support from an FPU scholarship from the Spanish Ministry of Education, and J. A. Sáez, from EC under FP7, Coordination and Support Action, Grant Agreement Number 316097, ENGINE European Research Centre of Network Intelligence for Innovation Enhancement (http://engine. pwr.wroc.pl/). We are thankful to Dr. Antonio D. Masegosa from the University of Deusto, Spain, for suggesting the use of video sequences for visualizing the convergence of dynamic optimization algorithms, which has proven very successful for this purpose.

\section{Bibliography}

D. A. Armstrong. factorplot: Improving presentation of simple contrasts in generalized linear models. The R Journal, 5(2):4-15, 2013. [p90]

T. Bartz-Beielstein, M. Chiarandini, L. Paquete, and M. Preuss, editors. Experimental Methods for the Analysis of Optimization Algorithms. Springer-Verlag, Berlin Heidelberg, 2010. [p89]

C. M. Bishop. Pattern Recognition and Machine Learning. Information Science and Statistics. Springer, 2006. [p100]

J. Branke. Memory enhanced evolutionary algorithms for changing optimization problems. In IEEE Congress on Evolutionary Computation (CEC), pages 1875-1882, 1999. [p96]

J. Branke. Evolutionary Optimization in Dynamic Environments. Genetic Algorithms and Evolutionary Computation (Book 3). Kluwer Academic Publishers, MA, USA, 2001. [p96]

M. Burda. paircompviz: An R Package for Visualization of Multiple Pairwise Comparison Test Results, 2014. URL https://www. bioconductor.org/packages/release/bioc/html/paircompviz.html. R package version 1.0.0. [p89]

C.-C. Chang and C.-J. Lin. LIBSVM: A Library for Support Vector Machines, 2001. URL http: //www. csie . ntu.edu. tw/ cjlin/libsvm. [p100]

M. Coffin and M. J. Saltzman. Statistical analysis of computational tests of algorithms and heuristics. INFORMS Journal on Computing, 12(1):24-44, 2000. [p89]

W. W. Cohen. Fast effective rule induction. In Proceedings of the Twelfth International Conference on Machine Learning, pages 115-123. Morgan Kaufmann Publishers, 1995. [p100]

C. Cruz, J. R. González, and D. A. Pelta. Optimization in dynamic environments: A survey on problems, methods and measures. Soft Computing, 15(7):1427-1448, 2011. [p96]

I. G. del Amo and D. A. Pelta. SRCS: A technique for comparing multiple algorithms under several factors in dynamic optimization problems. In E. Alba, A. Nakib, and P. Siarry, editors, Metaheuristics for Dynamic Optimization, volume 433 of Studies in Computational Intelligence, pages 61-77. SpringerVerlag, Berlin Heidelberg, 2013. [p90, 91]

I. G. del Amo, D. A. Pelta, J. R. González, and A. D. Masegosa. An algorithm comparison for dynamic optimization problems. Applied Soft Computing, 12(10):3176-3192, 2012. [p90, 96, 99] 
J. Demšar. Statistical comparisons of classifiers over multiple data sets. Journal of Machine Learning Research, 7:1-30, 2006. [p89]

J. Derrac, S. García, D. Molina, and F. Herrera. A practical tutorial on the use of nonparametric statistical tests as a methodology for comparing evolutionary and swarm intelligence algorithms. Swarm and Evolutionary Computation, 1(1):3-18, 2011. [p89]

M. J. Eugster, F. Leisch, and C. Strobl. (Psycho-)analysis of benchmark experiments: A formal framework for investigating the relationship between data sets and learning algorithms. Computational Statistics \& Data Analysis, 71:986-1000, 2014. [p89]

S. García, D. Molina, M. Lozano, and F. Herrera. A study on the use of non-parametric tests for analyzing the evolutionary algorithms' behaviour: A case study on the CEC'2005 special session on real parameter optimization. Journal of Heuristics, 15(6):617-644, 2009. [p89]

S. García, A. Fernández, J. Luengo, and F. Herrera. Advanced nonparametric tests for multiple comparisons in the design of experiments in computational intelligence and data mining: Experimental analysis of power. Information Sciences, 180(10):2044-2064, 2010. [p89]

M. Graczyk, T. Lasota, Z. Telec, and B. Trawiński. Nonparametric statistical analysis of machine learning algorithms for regression problems. In R. Setchi, I. Jordanov, R. Howlett, and L. Jain, editors, Knowledge-Based and Intelligent Information and Engineering Systems, volume 6276 of Lecture Notes in Computer Science, pages 111-120. Springer-Verlag, Berlin Heidelberg, 2010. [p89]

K. Hornik, C. Buchta, and A. Zeileis. Open-source machine learning: R meets Weka. Computational Statistics, 24:225-232, 2009. [p100]

IBM Corp. IBM SPSS Statistics for Windows, Version 21. Armonk, NY, 2012. URL http://www01.ibm.com/software/analytics/spss/products/statistics/. [p89]

I. Kononenko and M. Kukar. Machine Learning and Data Mining: Introduction to Principles and Algorithms. Horwood Publishing Limited, 2007. [p101]

M. Lichman. UCI Machine Learning Repository, 2013. URL http://archive.ics. uci.edu/ml. [p100]

D. Meyer, E. Dimitriadou, K. Hornik, A. Weingessel, and F. Leisch. e1071: Misc Functions of the Department of Statistics (e1071), TU Wien, 2014. URL https: //CRAN. R-project. org/package=e1071. $\mathrm{R}$ package version 1.6-4. [p100]

D. Nettleton, A. Orriols-Puig, and A. Fornells. A study of the effect of different types of noise on the precision of supervised learning techniques. Artificial Intelligence Review, 33:275-306, 2010. [p102]

D. Shilane, J. Martikainen, S. Dudoit, and S. J. Ovaska. A general framework for statistical performance comparison of evolutionary computation algorithms. Information Sciences, 178(14):2870-2879, 2008. [p89]

M. Still. The Definitive Guide to ImageMagick. Apress, NY, 2005. [p95]

P. J. Villacorta. SRCS: Statistical Ranking Color Scheme for Multiple Pairwise Comparisons, 2015. URL https: //CRAN.R-project.org/package=SRCS. R package version 1.1. [p90]

D. R. Wilson and T. R. Martinez. Improved heterogeneous distance functions. Journal of Artificial Intelligence Research, 6:1-34, 1997. [p100]

I. H. Witten and E. Frank. Data Mining: Practical Machine Learning Tools and Techniques. Morgan Kaufmann, San Francisco, 2nd edition, 2005. [p100]

$\mathrm{X}$. Zhu and X. Wu. Class noise vs. attribute noise: A quantitative study. Artificial Intelligence Review, 22: 177-210, 2004. [p100, 102]

Pablo J. Villacorta

Department of Computer Science and Artificial Intelligence,

University of Granada,

ETSIIT, C/Periodista Daniel Saucedo Aranda s/n, 18071 Granada, Spain

pjvi@decsai.ugr.es

José $A$. Sáez

ENGINE Centre,

Wrocław University of Technology,

Wybrzeże Wyspiańskiego 27, 50-370 Wrocław, Poland

jose.saezmunoz@pwr.edu.pl 